\title{
A Study on the Influence of Artificial Intelligence Research on the Development of Information Security Research
}

\author{
Tai-hoon $\mathrm{Kim}^{1}$ \\ ${ }^{1}$ Research Professor, The Academic Affairs Office, Glocal Campus of Konkuk University, Korea, \\ taihoonn@kku.ac.kr
}

\begin{abstract}
In order to combat the growing complexity of cybercrime, more intelligent and effective cybersecurity defense methods must be developed. As a result, a defense system can respond in real time to more sophisticated attacks. Researchers and practitioners should be familiar with current cyber security methods in order to assist in the solution of problems (Cybersecure). Artificial intelligence is frequently employed in the fight against cybercrime. Methods of combating cybercrime that make use of artificial intelligence, on the other hand, are uncommon. A total of 131 articles from two major databases are used to close this knowledge gap (ACM digital library and IEEE Xplore). The articles were mapped both quantitatively and qualitatively, according to the results. When it comes to fighting cybercrime and intrusion detection, artificial intelligence has made a significant contribution. It has also been discovered that the complexity of computers, the time required for model training, and the number of false alarms can all be reduced. However, the domain varies greatly. A common tool in intrusion detection and prevention systems, support vector machines (SVMs) are the most widely used type of machine.
\end{abstract}

Keywords: Artificial Intelligence, Machine Learning, Deep Learning, Security, Information

\section{Introduction}

Despite the fact that the majority[1] of people are intelligent, many are unable to comprehend and effectively solve problems. When it comes[2] to tasks that require dealing with operational errors and irregularities, artificial intelligence outperforms human abilities and skill. Artificial intelligence (AI) is required for the detection of human errors. The use of artificial intelligence[3] (AI) can assist businesses in protecting themselves against internet threats, detecting malware, setting[4] security standards, and improving their prevention and recovery procedures. This study demonstrates how artificial[5] intelligence technology and applications can assist with cybersecurity. Creating systems that are constantly[6] on the lookout for unauthorised network access, modified systems, and data is the ultimate goal of computer security research. Threat and vulnerability assessments $(\mathrm{T} \& \mathrm{~V})$ are a procedure that includes safeguards[7] for network and application security, privacy, and control, as well as other measures. Because of technological advancement, new risks emerge that change at a rapid pace. As a result, information and communications technology (ICT) advances. Over the last few years, the use of artificial intelligence (AI)[8] has increased dramatically, affecting virtually every aspect of life. Artificial intelligence is interested in the application of science and technology to the creation of automated machines.

AI is used in a variety of applications including games, production, and healthcare, in addition to

Received: July 16, 2021; $1^{\text {st }}$ Review Result: September 01, 2021; $2^{\text {nd }}$ Review Result: October 16, 2021 Accepted: November 30, 2021 
language processing[9], play, and production. Despite artificial intelligence's offensive and defensive capabilities, these information security advantages are still readily apparent. The similarities between industries that were previously thought to be distinct, such as leak detection and system security, have been demonstrated. The lack of preparedness of many businesses and organisations for increasingly sophisticated cyber-attacks[10] is a source of concern for many people. It is expected that the solution will be able to cover 85-95[11] percent of endpoints using advanced heuristics and signature-based solutions. A few large players have transformed cybercrime into a multi-billion-dollar industry from a billion-dollar industry that was dominated by a few large players. Researchers estimate that malware developers' return on investment (ROI)[12] is 1.425 percent (ROI). As long as there is significant trade, there will be an increase in the demand for improved information security. Increasingly popular internet usage has given rise to new industries[13] such as information security and artificial intelligence (see [Fig. 1]). The hacker will receive a monetary reward. In order to be beneficial to individuals, organisations, and governments, malicious software must evolve. Phishing, password attacks, and other forms of cybercrime fall under this category[14]. This virus has proven to be impervious to conventional safety measures.

Information security has been enhanced with the introduction of artificial intelligence (AI). Not only in emergencies, but also in a variety of other[15] situations, artificial intelligence systems can be of assistance. The automatic categorization and sorting of events and threats eliminates the need for technicians to perform time-consuming tasks[16]. This study investigates the various threats to information security as well as the history of artificial intelligence in the field of information security. The Internet, among other advances[17] in information and communications technology, has aided in both personal and professional development. People from all over the world can communicate with one another through the Internet. It encourages communication and interaction, which are both necessary for human development. Despite its numerous advantages, it has one significant drawback. It is becoming increasingly difficult to "touch"[18] information systems due to the increasing reliance on third-party and/or cloud-based data storage and applications. In the current cloud infrastructure, there are three layers, as an example. Vulnerabilities[19] in programmers and service providers can be found at every level of the stack. Attack each layer one by one. Detecting cybercrimes such as identity theft or financial fraud can be difficult. It is even more upsetting to note that the realization Criminals are now able to operate from any location in the world through distributed computing.

Because of this trend, businesses are becoming more vulnerable to cyberattacks, which is increasing the number of cyber-attacks. They are vulnerable to being countered or outperformed by artificial intelligence (AI) or smart agents. It is necessary to improve the intelligence[20], flexibility, and dependability of cyber-defence mechanisms in order to detect and counter cyber threats. An artificial intelligence system is being used to assist in the monitoring and combating of cyber-attacks and cybercrime. The use of information security techniques in artificial intelligence research is increasing, highlighting the importance of researchers and practitioners staying up to date on the latest developments. Some studies have summarized and discussed general information security issues; however, none have done so in an optimal manner when it comes to artificial intelligence applications. As a result of this, this article will examine current research on cyber-attack defence using artificial intelligence, with a particular emphasis on machine learning. This section discusses the application of artificial intelligence in the fight against high-level cyber-attacks (AI). The previous research and the current knowledge gap will be discussed in greater detail in the following sections.

\subsection{Research Methodologies and Objectives}

This study employed a thematic review of the literature. Relevant resources were discovered through searches in Google Scholars, Science Direct, Research Gates, and academia. In order to accomplish this, 
only papers published within the last five years were included in the study. In order to compile this list, the researchers examined the most innovative ideas that have been published in the last five years. Manuscripts containing more than five quotations were also considered for inclusion. Non-English documents, books, and patents were barred from participation. Our materials include relevant information in the abstract and conclusion sections, among other places. The primary key phrase in the paper was "artificial intelligence applications in information security," and it was used for confirmation control to identify the paper. As a result, only relevant papers were selected. For the purpose of addressing the paper's stated objectives, the application makes use of prior literature research and extrapolation of findings.

\subsection{Research Questions}

Specifically, the central question of this paper is "How can artificial intelligence be used to improve information security?". The main issue is that artificial intelligence (AI) is simply machine intelligence. In cyberspace, artificial intelligence can be very useful. Data mining for security purposes is concerned with identifying patterns in data.

\section{Extensive Literature Survey}

In order to maintain a high[21] level of digital intelligence, it is important to have secure internetconnected gadgets on hand (AI). Deep learning, natural language processing, and knowledge representation and reasoning (KRR) are some of the techniques that may be used to address today's cybersecurity challenges (ES). As an alternative, this research investigates alternative artificial intelligence techniques that are anticipated to have a major influence on the administration and development of smart cybersecurity services. As opposed to traditional security solutions, it is possible to automate and improve cybersecurity calculations through the use of artificial intelligence-based security intelligence modelling. We've also suggested prospective study areas to consider in order to assist frame future research into the topic. This website contains materials and guidance that are intended for academics and industry professionals who are involved in artificial intelligence technology.

The rapid rise of data science[22] over the last many years has resulted in significant advancements in information technology security. In order to comply with information security best practises, data extraction from various cyber-security sources and the development of a data-driven model are required. Data science is the examination and analysis of real-world data using a variety of scientific techniques, machine learning algorithms, procedures, and systems, among others. An in-depth look at the complicated process of data science for cyber defence, which includes data collection and analysis in order to stay up with data trends and build better security solutions, will be presented in this article. Smarter computing is enabled by data science solutions that make use of information security data. We will also examine and debate potential future research directions in this section. We also provide a platform for the development of sophisticated machine learning information security algorithms. We want to use data-driven, intelligent decision-making to help us prevent being hacked in the first place. Cyber-anomalies and cyber-attacks[23] continue to be a big source of concern in today's world. Machine learning can be used to address these difficulties.

Learning-based security approaches are effective when the risk and the data are considered together. Machine-based information security approaches such as "Cyberlearning" combine feature selection with empirical evaluation to provide a more complete picture of a threat. The models used for anomaly detection and cyber-attacks are binary classification models for anomaly detection and multiclass classification models for cyber-attacks. By combining 10 machines, we may construct a safety model (for example, naive Bayes, logistic regression, stochastic gradient descent, K-nearest neighbour, vector 
supporting machinery, decision tree, random forest, adaptive boost, and linear discrimination analysis). The security model is placed on top of a multi-layer artificial neural network to provide additional protection. For the evaluation of learning-based security models, a number of research have made use of the UNSW-NB15 and NSL-KDD datasets, respectively. One of the primary goals of our effort is to serve as a resource for our cybersecurity trials and discoveries.

The Internet of Things, information security[24], mobile data, business data, social media, and health data are all part of the Fourth Industrial Revolution's digital environment (4IR or Industry 4.0). This data requires a thorough understanding of artificial intelligence (AI), especially machine learning, in order to make sense of it (ML). Machine learning algorithms are available in a variety of flavours, including supervised, unsupervised, semi-supervised, and enhanced research. Because deep learning is a subset of machine learning, it is possible to analyse large volumes of data using this kind of analysis. Machine learning has the potential to enhance the intelligence and capabilities of an app. This research introduces machine learning algorithms and illustrates their application in cyber security, smart cities, health care, e-commerce, and agriculture, among other areas. In addition, we look at the difficulties that the results may have and possible remedies. This website is designed to serve as a resource for academics, industry, and policymakers alike in the field of artificial intelligence.

In recent years, the rapid proliferation[25] of computer networks, as well as the development of vital applications such as the Internet of Things, have raised public awareness of information security concerns (IT). If we do not put in place an effective intrusion detection system, our safety will be jeopardised. Machine learning might be used to develop an artificial intelligence-powered intrusion detection system. The Intrusion Detection Tree (Intrusted) is a type of intrusion detection system. First, we develop an intrusion detection model that incorporates all of the system's security attributes, and then we develop a more general model that incorporates the characteristics of the Intrusion Detection Tree. The reduced feature size in this model lowers the overall computation cost of the model. Our Intrusted model was validated using the datasets listed above. In this study, the outcomes are compared against a variety of commonly used learning approaches, such as naive Bayes classification, logistic regression, vector support, and the closest neighbour method.

During the last several years, artificial intelligence algorithms[26] have made significant progress, allowing smart phones to work successfully within computer systems. AI software frequently makes use of machine learning, deep learning, natural language processing, knowledge representation, and expert systems, among other techniques. This article summarises the concepts and AI-based modelling techniques that have been utilised in the development and manufacturing of intelligent mobile applications in order to increase human survival chances in a variety of scenarios. AI-powered healthcare applications, virtual assistants, and apps that combat public health concerns such as COVID19 are all investigated in this study. Finally, we explore a variety of issues and research objectives that have arisen as a result of our results. This website provides general information and help to app developers and researchers, with a particular emphasis on technological knowledge and assistance.

As the Internet continues to grow at a dizzying pace, hostile hacker[27] attacks are becoming increasingly common. There is a brief educational overview provided in this article, which also covers machine learning and deep learning. Time and/or temperature linkages are made for each of the methods described here. It is possible to increase cybersecurity with the help of machine learning and deep learning. It is possible to do research since data is essential for machine learning and deep learning.

\section{Research Gap}

\subsection{AI for Information Security}

Information security (InfoSec) is a major source of concern[28] for information and communications 
technology (ICT) researchers. The issue has been addressed in a number of previous studies by incorporating technological advances such as malware detectors, intrusion detection and prevention systems, advanced firewall configurations, and data encryption[29] algorithms. Many studies have found it beneficial to concentrate on human behaviour when solving information security problems, but others have found it to be insufficient. For example, consider the vast majority of businesses that deal with large amounts of data and thus necessitate a high degree of automation. The integration of human, technological, and political elements into organisational safety activities is essential. In the past, technology has concentrated on preventing threats by emphasising[30] physical security devices and pre-determined algorithmic structures. For example, the very first antivirus scanning systems looked for viruses that had a specific bit signature in their code. Assumption: All viruses have a structure and pattern that are similar to one another. These algorithms and signatures have no way of being reversed. Despite the fact that it is updated on a daily basis, this strategy is lacking in sophistication and frequency (or when the device is connected to the Internet).

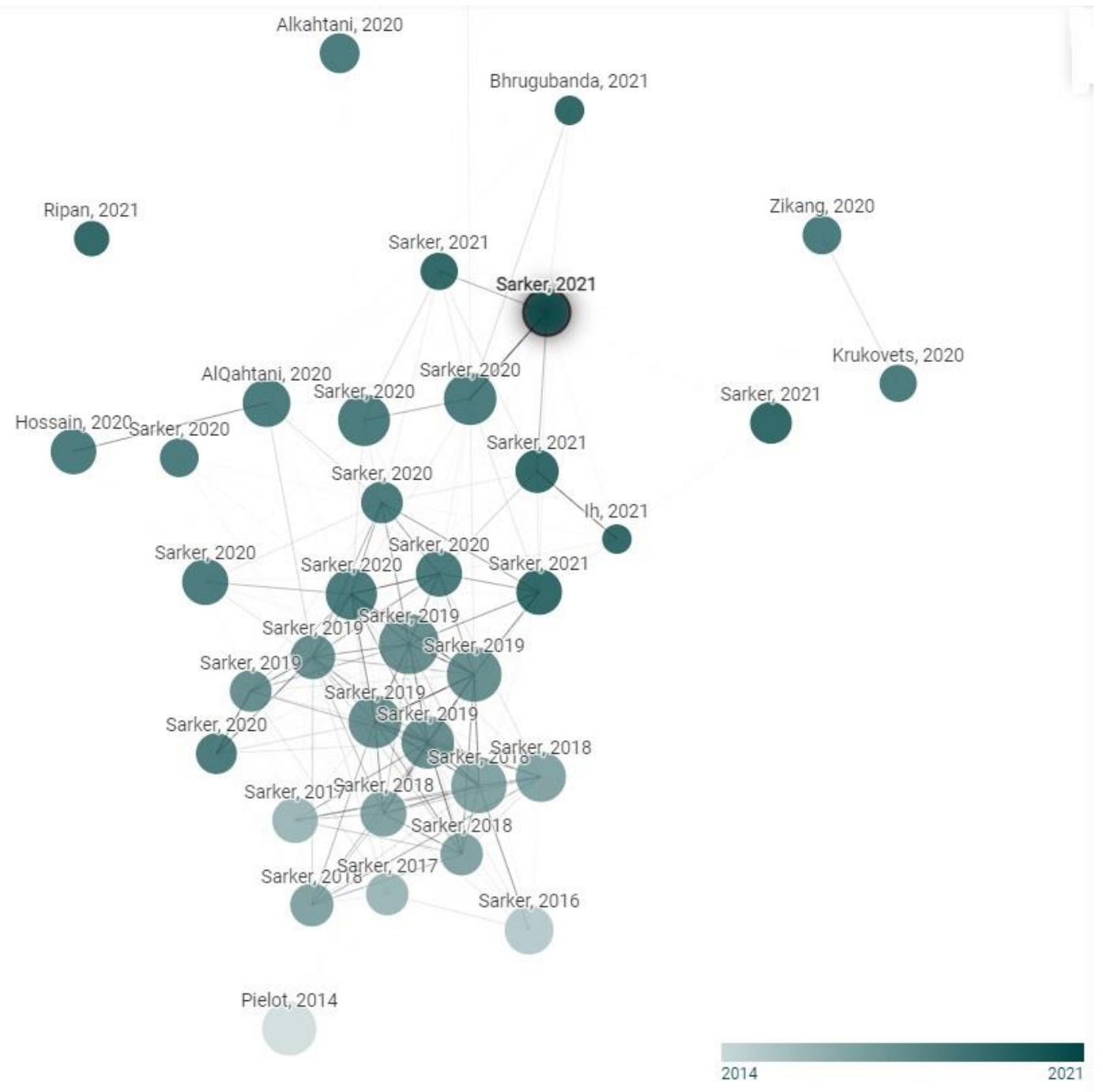

[Fig. 1] Showing the Extensive Literature Survey of AI for Information Security

In order to detect and [31] mitigate malware attacks, it is no longer necessary to use a signature. In order to combat this, systems for detecting and preventing malicious AI activity must be developed that are both efficient and effective. Furthermore, they establish useful standards and mechanisms for controlling and preventing cyber-attacks. Researchers frequently struggle to determine which artificial 
intelligence approach is the most efficient, as well as how it will affect information security in the future. While it is unquestionably true that artificial intelligence can help to secure the workplace, the claims made are unsubstantiated and based on speculation. Previous research compared the performance of the invention to existing methods or examined a collection of systems, with the invention's performance being compared to existing methods in the vast majority of cases. The selection distortion in each of the three scenarios is approximately the same. Annotated literature summaries are required in order to identify new research questions, challenges, and directions for future research.

\subsection{AI Applications that Contribute to enhancing the Information security}

This research axis will look into how Artificial Intelligence can be used to improve information security in the future. A growing amount of data is being generated in today's world, and this data is being stored and received via the Internet. The transmission of data across a network is also required in the fight against cybercrime, as proper information transmission is critical[32] in the fight against cybercrime. Criminals are increasingly turning to cybercrime, which is wreaking havoc on the internetbased community. Artificial intelligence and information security are broad concepts that include risk mitigation, revenue growth, and the detection of cyber threats. It will be easier to detect[33] and respond to threats and malware if information security powered by artificial intelligence can analyse historical cyber-attack data, which will be accomplished through data mining. When it comes to detecting malicious malware, artificial intelligence (AI) may prove to be more effective and efficient than humans. The use of artificial intelligence in conjunction with security information and event management is used within the organisation[34] to assist security analysts in identifying threats in the network. "The lower the costs, the more quickly the data breach was discovered, and vice versa. The severity of criminals and malicious attacks this year has resulted in the lengthier time it takes to resolve a violation this year.

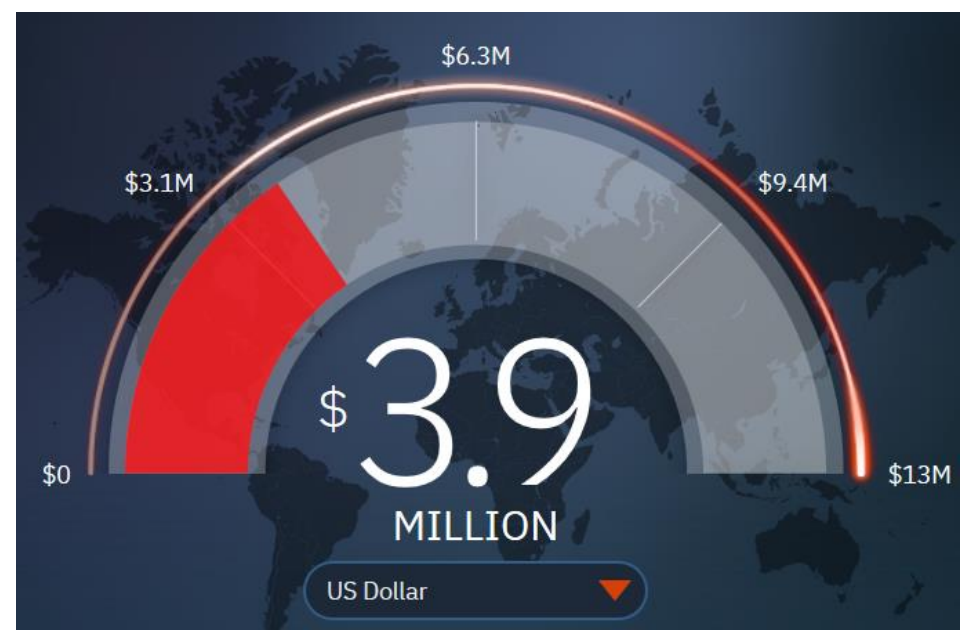

[Fig. 2] Showing the Amount of Data Breach

It is possible to improve an organization's ability to detect and respond to hazards through the use of safety automation and intelligent orchestration, to lessen the severity of a violation 'AI applications are used in a variety of applications, including SIEMs[35], spam filters, secure user authentication, and incident hacking prediction. These applications are programmed to detect malware based on the behaviour of the user in previous sessions. Either it is, or it isn't. Either it is, or it isn't. According to IBM, automating security solutions lowers the overall cost of a data breach on an international scale. Companies that have never done so in the past Compared to non-automated organisations; the cost of a security breach was 95 percent higher in automated organisations ""Without a doubt." When automatic 
security solutions are not implemented, data breaches in the Middle East can result in significant financial losses (see [Fig. 2]).

\subsection{Applications of Expert Systems in Information Security}

Expert systems are well-known artificial intelligence tools. They are made up of a variety of software packages that provide assistance to customers or other software packages[36] in their search for answers. This system contains information that is relevant to a specific application. A reasoning system that incorporates the logic allows users to obtain answers based on their input and other relevant environmental information.

\subsection{ML applications in Artificial Intelligence}

Instead of anticipating every possible scenario, a computer can learn by studying examples of previous data. Supervised and unattended machine learning are the two most common machine learning methods. When a relevant identified instance dataset is available, a supervised study is carried out in conjunction with it. Being able to accurately predict values or categorise inputs is beneficial in decisionmaking and prediction. The use of uncontrolled learning is employed in the absence of a labelled dataset. Unattended learning techniques, such as clustering, take advantage of people's natural tendency to group themselves into categories in the same way.

Clustering is a technique for identifying data patterns. For the classification and supervision of unclassified data, cluster analysis[37] is a widely used technique. In order to combat cyber-threats, a constant and immediate response is required. When it comes to applying artificial intelligence to cybersecurity, deep training, which does not require prior training or expert classification, is critical. We will look at deep learning, which is a crucial subset of machine learning, and demonstrate how it can be used to improve cybersecurity. After that, we'll discuss deep learning and demonstrate how it can make a significant difference. One of the objectives of the study was to evaluate machine learning-based cybersecurity techniques. Machine learning was used in this study to identify potential participants. Malware, spam, and other intrusions are all classified as "intrusions" and fall under this category. There are numerous shortcomings to machine-based solutions, a variety of approaches to cyber-education

\subsection{Deep Learning Applications in Information security}

Unbundling data is a significant challenge for the majority of cybersecurity research organisations. However, corporate security professionals have discovered that even companies with extensive internal expertise can use classified data to train machine learning models, despite the fact that the return to confidentiality is often associated with scarcity. Unbalanced[38] datasets, manual categorization, and other factors all play a role in this. Researchers have recently developed a framework for cybersecurity research based on the three traditional learning methods of supervised, semi-subjective, and unconsidered learning. They used the three traditional learning methods of supervised, semi-subjective, and unconsidered learning.

\subsection{Data Mining Applications to Enhance Information Security}

Patterns and trends are discovered by mining large databases. Data mining is the process of searching through large amounts of data for hidden patterns. This chapter introduces machine learning, databases, statistics, specialised systems, visualisations, high-performance computing, rough sets, neural networks, and knowledge representation (Knowledge Representation and Representation of Knowledge). A host 
that collects data in a variety of ways aids in the collection of data (e.g., clustering, classification, link analysis, summarization, regression models, and sequence analysis). Here are a few examples of data mining in the context of cybersecurity[39]: It takes a variety of techniques to recognise and analyse unusual patterns and behaviours. When a virus is detected, a connection is established. Through the use of this approach, an extensive number of distinctive profiles emerge, each of which can be used to identify cyber-attacks. Forecasts are made based on information gathered in order to identify potential future attacks. Viruses are discovered through link analysis. Unusual methods are employed in order to detect and analyse anomalous behaviour and patterns. Prediction models can be used to predict attacks in the future.

\subsection{Recommended Solutions}

Prior research was used to analyse and describe literary works in this study, which was conducted after the fact. Unsupervised machine learning, deep learning, and data mining have all been discovered to be used for intrusion detection, malware analysis, and spam detection, among other applications. A number of flaws in cybersecurity education were discovered as a result of the findings, including the fact that all vulnerabilities are counter attackable and difficult to automate. It is possible to improve the performance of identification by using machine-readable workbooks that contain a variety of threat indicators. At this point in the development process, it is impossible to assess the security of machine learning. If current and promising adverse learning approaches are taken into consideration, it is possible to make significant progress. Using deep learning and other unattended learning algorithms to train cybersecurity professionals is becoming increasingly popular in the field.

There are numerous advantages to using cyber-security-based security systems. It is possible to significantly reduce the amount of time spent by humans when using deep learning algorithms in information security to detect suspicious behaviour patterns. It is necessary to employ data mining strategies and algorithms in order to detect malware from a variety of data sources. Data that is frequently used the requirements for anomaly, abuse, and hybrid detection differ depending on the data mining strategy being used to detect them. These approaches, which allow for the use[40] of any data mining algorithm, each have their own set of advantages and disadvantages, but they are not mutually exclusive. Decision Tree Learning, Naive Bayes Classifier, KNearest Neighbor, and Support Vector Machine are some of the malware detection algorithms that are available. These approaches are ineffective. The complexity of the algorithms, the memory requirements, and the workflow all contribute to the difficulty. Malware can now be identified and classified using data-mining algorithms. On the other hand, it is necessary to develop new and more efficient malware detection and classification algorithms.

\section{Review Results}

In addition, the year of publication, the author's address, and the country of the publishing press have been entered. Each article also included a description of an artificial intelligence method and a security application. Studies examining ways to improve existing methods were also reviewed and summarised in this report. The findings of the study were as follows, in the [Fig. 3].

According to the findings of the study, the number of publications on methods for ensuring artificial intelligence information security has increased significantly in recent years (see [Fig. 2]). After 2015, artificial intelligence in cybersecurity gained traction. Between 2008 and 2015, primary studies accounted for 26 percent of all articles reviewed. The steepest incline occurred between 2016 and 2018. There has been a nearly twofold increase in the number of publications in the area between 2015 and 
2017. In 2018, 57 studies were published, which is nearly double the number of studies published in 2017.

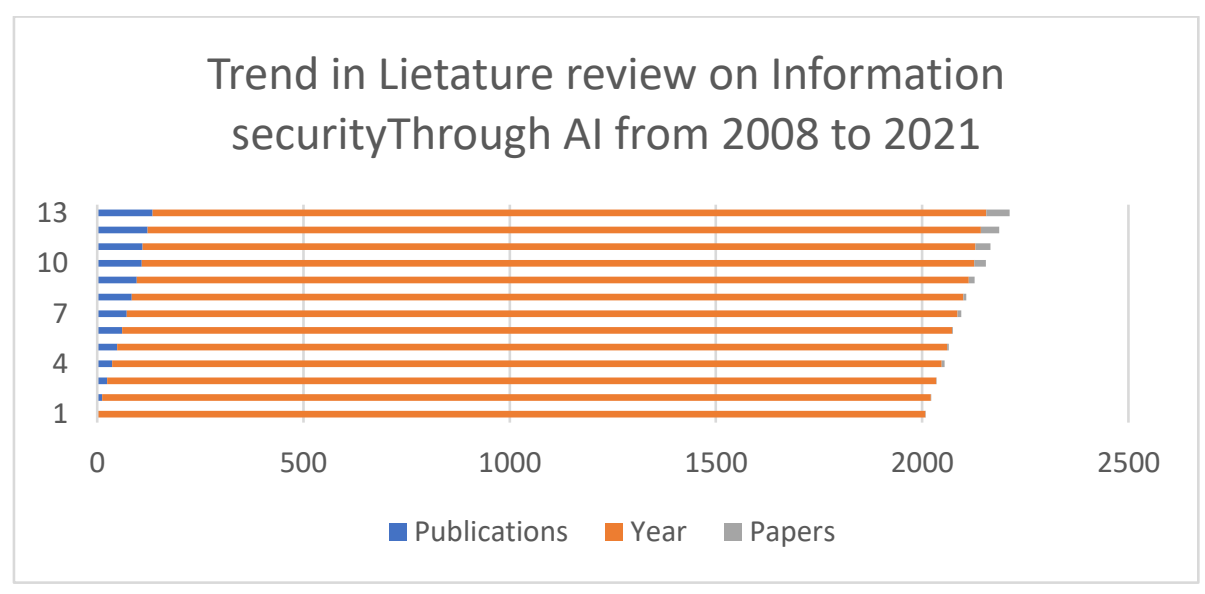

[Fig. 3] Trends in Literature Review on Information Security from 2008 to 2021

This investigation also revealed an asymmetry between publishers and distribution channels. 32 articles from the IEEE Access journal and 26 articles from the IEEE Information Forensic and Security journal were among the 131 journal articles reviewed (59 publications). [Fig. 3] depicts the distribution of publications according to the publishing house that published them. As illustrated in this illustration, vendors must contend with more than two publications. The IEEE Transactions on Information Theory and the IEEE Journal on Selected Areas in Communications, which are both published by the IEEE, have the second and third highest number of papers, respectively. They each conducted five separate studies on their own time. Only three studies were made available by the other publishers. Geographical location has also been shown to influence domain research. According to the publications of the corresponding authors, the vast majority of the studies were carried out in Asian countries. Asian authors contributed to 52 percent of the 131 articles in this collection. [Fig. 5] All of them were published in South America and Africa. The vast majority of works are published in China, with the United States coming in a close second. [Fig. 4] depicts the global distribution of articles studied by country as depicted in the data.

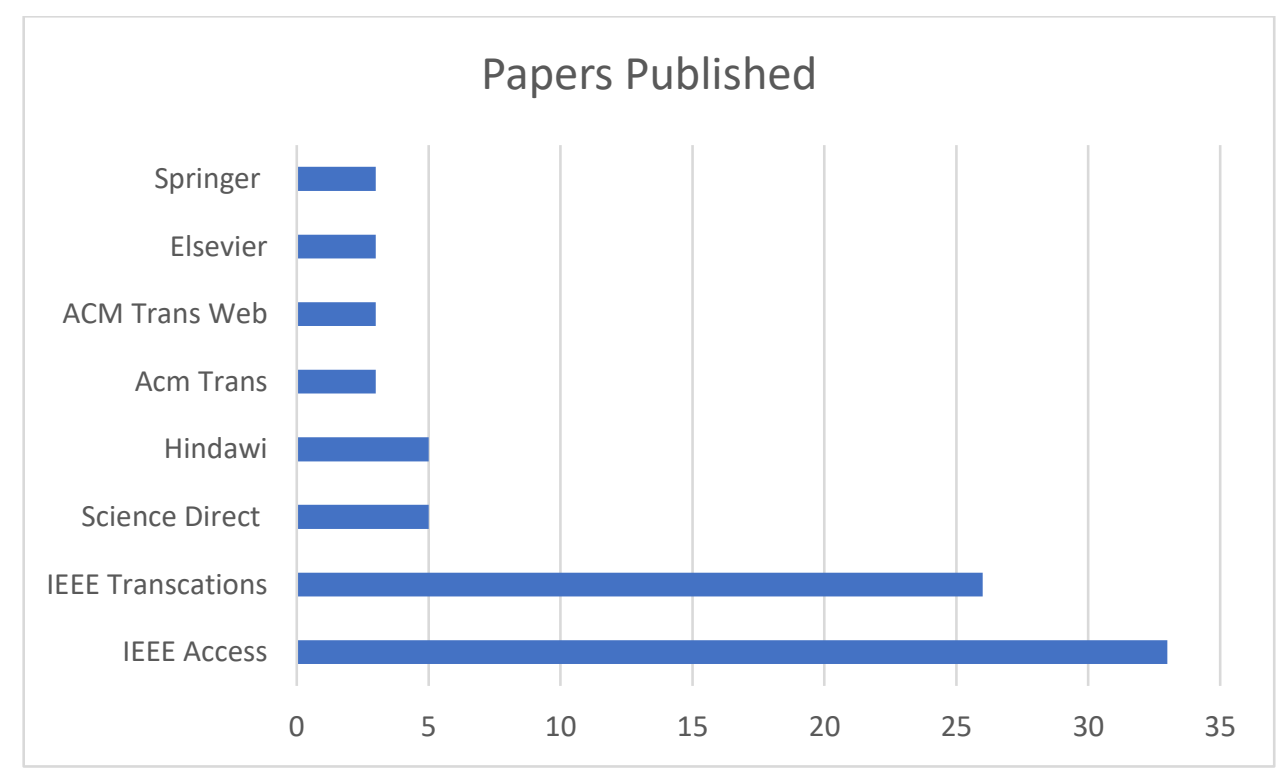

[Fig. 4] Showing the Published Articles and their Frequency 


\section{Distribution of Publications by country of Authors}

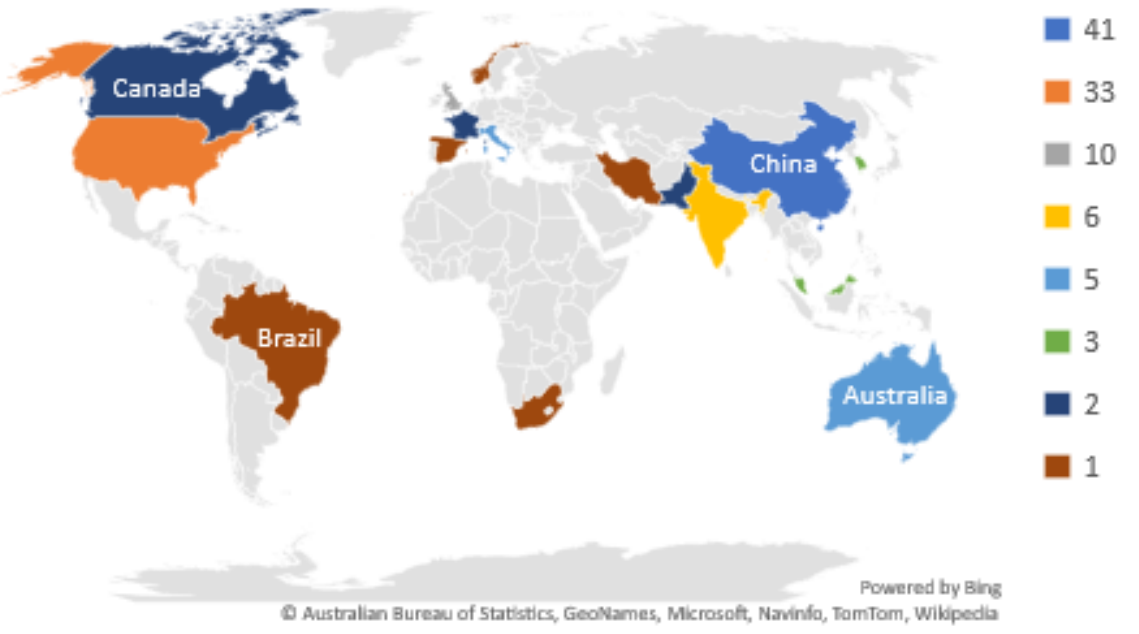

[Fig. 5] Showing the Research of Ai on Cyber Security by Country Wise Distribution

\subsection{AI Algorithms in Information Security}

Many different algorithms have been identified in the many studies used in the review. Artificial Neural Networks were the most popular algorithms, which incorporated the following: (ANN)[41-44]. CNN, DTs, and DTs, along with K-means, K-nearest neighbours (KNN), and AdaBoost, SVM [Table 1].

Covert channels and security games, fingerprint vitality detection, biometric (including facial verification), malicious webpages, cyberbullying, data injections, and risk data assessment were all mentioned in only two studies. Other topics covered included fingerprint vitality detection, biometric (including facial verification), malicious webpages, cyberbullying, data injections, and risk data assessment. There were 47[45-46] studies conducted on this subject (i.e., 36 percent). In order to help visualise how various algorithms have been applied, a number of methods have been mapped to a number of different application fields. Malware, viruses, and phishing are all used in more than half of all IDPS attacks (i.e., uses a combination of two or more AI algorithms). Other research has discovered evidence against ensembles in the areas of encryption, service denial, imagery, and CAPTCHA, among other things.

[Table 1] Showing the Ai Algorithms on Cyber Security with Respective to Mechanism

\begin{tabular}{|c|c|c|c|c|c|c|c|}
\hline S. No & Algorithm & \multicolumn{6}{|c|}{ Mechanism } \\
\hline 1 & Decision Tree & Intrusion & 18 & 2 & 9 & 4 & 1 \\
\hline 2 & RNN & Encryption & 1 & 3 & 9 & 4 & 6 \\
\hline 3 & Random Forest & Imaging & 2 & 5 & 9 & 5 & 1 \\
\hline 4 & Q Learning & Captcha & 4 & 7 & 9 & 4 & 7 \\
\hline 5 & K Means & Phishing & 5 & 8 & 5 & 6 & 3 \\
\hline 6 & Artificial Neural Network & Malware & 67 & 9 & 3 & 4 & 4 \\
\hline 7 & $\mathrm{KNN}$ & Traffic & 78 & 0 & 3 & 4 & 4 \\
\hline 8 & Support Vector Machines & Traffic Classification & 9 & 3 & 3 & 4 & 3 \\
\hline 9 & Convolutional Neural Network & DOS & 5 & 3 & 4 & 22 & 1 \\
\hline 10 & AdaBoost & Ransomware & 4 & 4 & 6 & 4 & 3 \\
\hline 11 & XgBoost & intrusion Detection & 3 & 3 & 6 & 4 & 5 \\
\hline 12 & VGG-16 & Certifications & 3 & 2 & 6 & 4 & 1 \\
\hline 13 & LSTM & Others & 18 & 5 & 9 & 4 & 1 \\
\hline
\end{tabular}




\section{Conclusions}

The following are the most significant findings of this study: Data, information, and systems are all safeguarded by information security. Machine learning, deep learning, data mining, and expert systems are some of the ways that artificial intelligence (AI) may assist with cyber security. Data mining may be employed in the development and support of information security systems.

\section{Acknowledgement}

This paper was supported by Konkuk University in 2021 .

\section{References}

[1] Z. Brakerski, C. Gentry, V. Vaikuntanathan, (Leveled) Fully Homomorphic Encryption without Bootstrapping, Proceedings of the 3rd Innovations in Theoretical Computer Science Conference, (2012), January 8-10; Cambridge, Massachusetts, DOI: https://doi.org/10.1145/2090236.2090262

[2] M. Ben Neria, N.-S. Yacovzada, I. Ben-Gal, A Risk-Scoring Feedback Model for Webpages and Web Users Based on Browsing Behavior, ACM Transactions on Intelligent Systems and Technology, (2017), Vol.8, No.4, pp.1-21, DOI: https://doi.org/10.1145/2928274

[3] I. A. Gheyas, A. E. Abdallah, Detection and prediction of insider threats to cyber security: a systematic literature review and meta-analysis, Big Data Analytics, (2016), Vol.1, No.6, p.1-29, DOI: https://doi.org/10.1186/s41044-016-0006-0

[4] Z.-M. Zhang, F.-F. Shi, Y.-L. Wan, Y. Xu, F. Zhang, H.-S. Ning, Application progress of artificial intelligence in military confrontation, Chinese Journal of Engineering, (2020), Vol.42, No.9, pp.1106-1118, DOI: 10.13374/j.issn20959389.2019.11.19.001

[5] N. S. Aljurayban, A. Emam, Framework for cloud intrusion detection system service, 2015 2nd world symposium on web applications and networking (WSWAN), (2015), March 21-23; Sousse, Tunisia.

[6] A. Amberkar, P. Awasarmol, G. Deshmukh, P. Dave, Speech recognition using recurrent neural networks, 2018 international conference on current trends towards converging technologies (ICCTCT), (2018), March 1-3; Coimbatore, India.

[7] B. Bakhshi, H. Veisi, End to end fingerprint verification based on convolutional neural network, 2019 27th Iranian conference on electrical engineering (ICEE), (2019), April 30-May 2; Yazd, Iran.

[8] H. Bao, H. He, Z. Liu, Z. Liu, Research on information security situation awareness system based on big data and artificial intelligence technology. 2019 international conference on robots' intelligent system (ICRIS), (2019), June 1516; Haikou, China.

[9] H. Yang, Y. Jia, W.-H. Han, Y.-P. Nie, S.-D. Li, X.-J. Zhao, Calculation of network security index based on convolution neural networks, Artificial Intelligence and Security, (2019), July 26-28; New York, USA, pp.530-540, DOI: https://doi.org/10.1007/978-3-030-24271-8_47.

[10] Y. Zeng, Z. Qi, W. Chen, Y. Huang, X. Zheng, H. Qiu, Test: an end-to-end network traffic examination and identification framework based on spatio-temporal features extraction, Cryptography and Security, (2019), http://arxiv.org/abs/1908.10271

[11] Z. Dongmei, L. Jinxing, Study on network security situation awareness based on particle swarm optimization algorithm, Computers \& Industrial Engineering, (2018), Vol.125, pp.764-775, DOI: https://doi.org/10.1016 /j.cie.2018.01.006

[12] T. Gu, B. Dolan-Gavitt, S. Garg, BadNets: identifying vulnerabilities in the machine learning model supply chain, Cryptography and Security,(2017), arXiv:1708.06733

[13] H. Hong, M. Lee, K. Park, Convolutional neural network-based finger-vein recognition using nir image sensors, 
Sensors, (2017), Vol.17, No.6, DOI: https://doi.org/10.3390/s17061297

[14] W. Hu, Y. Tan, Generating adversarial malware examples for black-box attacks based on gan, Machine Learning, (2017), http://arxiv.org/abs/1702.05983

[15] L. Kong, G. Huang, Y. Zhou, J. Ye, Fast abnormal identification for large scale internet traffic, Proceedings of the 8th international conference on communication and network security, (2018), November 2-4; New York, USA, DOI: https://doi.org/10.1145/3290480.3290498

[16] C. S. Kruse, B. Frederick, T. Jacobson, D. K. Monticone, Cybersecurity in healthcare: a systematic review of modern threats and trends, Technology and Health Care, (2017), Vol.25, pp.1-10, DOI: https://doi.org/10.3233/THC-161263

[17] M. Naderpour, J. Lu, G. Zhang, An intelligent situation awareness support system for safety-critical environments, Decision Support Systems, (204), Vol.59, pp.325-340, DOI: https://doi.org/10.1016/j.dss.2014.01.004

[18] Y. Shi, T. Li, L. Renfa, X. Peng, P. Tang, An immunity-based iot environment security situation awareness model, Journal of Computer and Communications, (2017), Vol.5, No.7, pp.182-197, DOI: https://doi.org/10.4236/ jcc. 2017.57016

[19] M. Tyworth, N. A. Giacobe, V. F. Mancuso, M. D. McNeese, D. L. Hall, A human-in-the-loop approach to understanding situation awareness in cyber defence analysis, EAI Endorsed Transactions on Security and Safety, (2013), Vol.13, No.2, pp.1-10, DOI: https://doi.org/10.4108/trans.sesa.01-06.2013.e6

[20] Z. J. Wang, R. Turko, O. Shaikh, H. Park, N. Das, F. Hohman, M. Kahng, D. H. Chau, CNN explainer: learning convolutional neural networks with interactive visualization, IEEE Transactions on Visualization and Computer Graphics, (2021), Vol.27, No.2, pp.1396-1406, DOI: https://doi.org/10.1109/TVCG.2020.3030418

[21] I. H. Sarker, M. H. Furhad, R. Nowrozy, AI-Driven Cybersecurity: An Overview, Security Intelligence Modeling and Research Directions, SN Computer Science, (2021), Vol.2, No.173, pp.1-18, DOI: https://doi.org/10.1007/s42979-02100557-0

[22] I. H. Sarker, A. S. M. Kayes, S. Badsha, H. AlQahtani, P. Watters, A. Ng, Cybersecurity data science: an overview from machine learning perspective, Journal of Big Data, (2020), Vol.7, No,41, pp.1-29, DOI: https://doi.org/ $10.1186 /$ s40537-020-00318-5

[23] I. H. Sarker, Cyber Learning: Effectiveness Analysis of Machine Learning Security Modelling to Detect CyberAnomalies and Multi-Attacks, Internet Things, (2021), Vol.14, No.100393, DOI: https://doi.org/10.1016/ j.iot.2021.100393

[24] I. H. Sarker, Machine Learning: Algorithms, Real-World Applications and Research Directions, Sn Computer Science, (2021), Vol.2, No.160, pp.1-21, DOI: https://doi.org/10.1007/s42979-021-00592-x

[25] I. H. Sarker, Y. B. Abushark, F. Alsolami, A. I. Khan, IntruDTree: A Machine Learning Based Information Security Intrusion Detection Model, Symmetry, (2020), Vol.12, No.5, pp.1-15, DOI: https://doi.org/10.3390/sym12050754

[26] I. H. Sarker, M. M. Hoque, M. K. Uddin, T. Alsanoosy, Mobile Data Science and Intelligent Apps: Concepts, AIBased Modelling and Research Directions, Mobile Networks and Applications, (2021), Vol.26, pp.285-303, DOI: https://doi.org/10.1007/s11036-020-01650-z

[27] Y. Xin, L. Kong, Z. Liu, Y. Chen, Y. Li, H. Zhu, M. Gao, H. Hou, C. Wang, Machine Learning and Deep Learning Methods for Cybersecurity, IEEE Access, (2018), Vol.6, pp.35365-35381, DOI: 10.1109/ACCESS.2018.2836950

[28] Azzah Kabbas, Atheer Alharthi, Asmaa Munshi, Artificial Intelligence Applications in Cybersecurity, International Journal of Computer Science and Network Security, (2020), Vol.20, No.2, pp.120-124, WEB: http://paper.ijcsns.org/ 07 book/202002/20200216.pdf

[29] G. Giacinto, R. Perdisci, M. Del Rio, F. Roli, Intrusion detection in computer networks by a modular ensemble of oneclass classifiers, Information Fusion, (2008), Vol.9, No.1, pp.69-82, DOI: 10.1016/j.inffus.2006.10.002

[30] E. Menahem, A. Shabtai, L. Rokach, Y. Elovici, Improving malware detection by applying multi-inducer ensemble, Computational Statistics \& Data Analysis, (2009), Vol.53, No.4, pp.1483-1494, DOI: 10.1016/j.csda.2008.10.015

[31] R. Leszczyna, A review of standards with cybersecurity requirements for smart grid, Computers \& Security, (2018), 
Vol.77, pp.262-276, DOI: 10.1016/J.COSE.2018.03.011

[32] L. Coventry, D. Branley, Cybersecurity in healthcare: A narrative review of trends, threats and ways forward, Maturitas, (2018), Vol.113, pp.48-52, DOI: 10.1016/j.maturitas.2018.04.008

[33] P. Brereton, B. Kitchenham, D. Budgen, M. Turner, M. Khalil, Lessons from applying the systematic literature review process within the software engineering domain, Journal of Systems and Software, (2007), Vol.80, No.4, pp.571-583, DOI: $10.1016 /$ j.jss.2006.07.009

[34] S. Ahmed, Y. Lee, S.-H. Hyun, I. Koo, Feature selection-based detection of covert cyber deception assaults in smart grid communications networks using machine learning, IEEE Access, (2018), Vol.6, pp.27518-27529,2018, DOI: 10.1109/ACCESS.2018.2835527

[35] Y. Gao, Y. Liu, Y. Jin, J. Chen, H. Wu, A novel semi supervised learning approach for network intrusion detection on cloud based robotic system, IEEE Access, (2018), Vol.6, pp.50927-50938, DOI: 10.1109/ACCESS.2018.2868171

[36] J. Yu, B. Zhang, Z. Kuang, D. Lin, J. Fan, IPrivacy: Image privacy protection by identifying sensitive objects via deep multi-task learning, IEEE Transactions on Information Forensics and Security, (2017), Vol.12, No.5, pp.1005-1016, DOI: 10.1109 /TIFS.2016.2636090

[37] B. Bayar, M. C. Stamm, Constrained convolutional neural networks: A new approach towards general purpose image manipulation detection, IEEE Transactions on Information Forensics and Security, (2018), Vol.13, No.11, pp.26912706, DOI: 10.1109/TIFS.2018.2825953

[38] P. S. Deng, J.-H. Wang, W.-G. Shieh, C.-P. Yen, C.-T. Tung, Intelligent automatic malicious code signatures extraction, IEEE 37th Annual 2003 International Carnahan Conference onSecurity Technology, (2003), October 14-16; Taipei, Taiwan, DOI: 10.1109/CCST.2003.1297626

[39] N. Aldaraani, Z. Begum, Understanding the impact of ransomware: A survey on its evolution, mitigation and prevention techniques, 2018 21st Saudi Computer Society National Computer Conference (NCC), (2018), April 2526; Riyadh, Saudi Arabia, DOI: 10.1109/NCG.2018.8593029

[40] A. Vance, M. Siponen, S. Pahnila, Motivating IS security compliance: Insights from habit and protection motivation theory, Information \& Management., (2012), Vol.49, No.3-4, pp.190-198, DOI: 10.1016/J.IM.2012.04.002

[41] P. Dennis, A. Stuart, 12 risks that threaten human civilization: The case for a new risk category, Global Challenges Foundation, (2015), http://globalchallenges.org/wp-content/uploads/12-Risks-with-infinite-impact.pdf

[42] L. Lazic, Benefits from AI in Cyber Security, The 11th international Conference on Business Information Security, (2019), October 18; Belgrade, Serbia.

[43] M. F. AbRazak, N. B. Anuar, F. Othman, A. Firdaus, F. Afifi, R. Salleh, Bio-inspired for features optimization and malware detection, Arabian Journal of Science and Engineering, (2018), Vol.43, pp.6963-6979, DOI: https://doi.org/10.1007/s13369-017-2951-y

[44] D. Selma, C. Huseyin, A. Mustafa, Application of Artificial Intelligence Techniques to Combating Cyber Crimes: A Review, International Journal of Artificial Intelligence \&Applications, (2015), Vol.6, No.1, pp.21-39, DOI: 10.5121/ijaia.2015.6102

[45] A. M. Shamiulla, Role of Artificial Intelligence in Cyber Security, International Journal of Innovative Technology and Exploring Engineering, (2019), Vol.9, No.1, pp.4628-4630, DOI: 10.35940/ijitee.A6115.119119

[46] S. Sawyer, M. H. Jarrahi, Sociotechnical approaches to the study of information systems, Computing handbook, 3rd edition, Chapman and Hall, (2014) 